\title{
Ohtahara syndrome in a family with an ARX protein truncation mutation (c.81C $>$ G/p.Y27X)
}

\author{
Tod Fullston 1,2,7, Louise Brueton ${ }^{3,7}$, Tracey Willis ${ }^{4}$, Sunny Philip ${ }^{4}$, Lesley MacPherson ${ }^{5}$, Merran Finnis ${ }^{2}$, \\ Jozef Gecz ${ }^{1,2,6}$ and Jenny Morton ${ }^{\star 3}$
}

\begin{abstract}
Aristaless-related homeobox ( $A R X)$ gene mutations cause a diverse spectrum of disorders of the human brain, including lissencephaly, various forms of epilepsy and non-syndromic mental retardation. We have identified a novel mutation, c.81C $>$ G (p.Y27X), within the $A R X$ gene in a family with two affected male cousins. One of the boys was diagnosed with an early infantile epileptic encephalopathy also known as Ohtahara syndrome, whereas his cousin had been diagnosed with West syndrome (WS). Both patients have normal genitalia and neither have lissencephaly. The $A R X$ mutation identified is predicted to yield a severely truncated protein of only $\mathbf{2 6}$ amino acids and can be considered as a null mutation. Somewhat surprisingly, however, it does not yield the X-linked lissencephaly with ambiguous genitalia (XLAG) syndrome. We proposed that the ARX mRNA translation re-initiated at the next AUG codon at position c.121-123 (aa 41) and, thus, partly rescued these patients from XLAG. Our in vitro studies show that this $\mathrm{N}$-terminally truncated ARX protein (p.M41_C562) is detected by western immunoblot in lysates from cells transiently transfected with an $A R X$ over-expression construct containing the c.81C $>\mathrm{G}$ mutation. Although these findings widen the spectrum of clinical phenotypes because of mutations in the $A R X$ gene, they also emphasize the molecular pathogenetic effect of individual mutations as well as the effect of genetic background resulting in intrafamilial clinical heterogeneity for these mutations.
\end{abstract}

European Journal of Human Genetics (2010) 18, 157-162; doi:10.1038/ejhg.2009.139; published online 9 September 2009

Keywords: Ohtahara syndrome; burst suppression; ARX gene; West syndrome

\section{INTRODUCTION}

The ARX gene is located on the short arm of the $\mathrm{X}$ chromosome, and since its discovery in $2002,{ }^{1-3}$ it has been implicated in a number of different syndromes and non-syndromic mental retardation. ${ }^{4}$ Limited human and extensive mouse gene-expression studies show high levels of $A R X$ expression in the foetal brain as well as in some parts of the adult brain. In particular, $A R X$ expression is high in the neuronal precursors of the germinal matrix and ventricular zones at all stages of development. High levels of expression are also observed in the subventricular zone, the caudate nucleus, putamen, substantia nigra, corpus callosum, amygdala and hippocampus. ${ }^{1-3,5,6}$ This expression during early development and predilection for neuronal tissue suggests that ARX has a pivotal function in neurodevelopment.

We report a family with two male cousins (IV-1 and IV-2, Figure 1a), who were diagnosed with West syndrome (WS) and Ohtahara syndrome (OS), respectively. There is an additional family history of stillbirth, perinatal death and epilepsy in males, compatible with X-linked inheritance. An $A R X$ mutation, c.81C $>\mathrm{G}$ in exon 1, was identified in both cousins and their mothers. This mutation represents the first ARX protein truncation mutation that causes a non-malformation phenotype. This apparent lack of a severe malformation is probably because of the re-initiation of the translation from p.M41, allowing an N-terminally truncated, partially functional ARX protein to be produced.

\section{MATERIALS AND METHODS}

Patient data

The proband (IV-1) is the maternal first cousin of IV-2, as their mothers are sisters. Both mothers are of normal intelligence, in good health and have no history of seizures. There are no surviving males in their mother's or grandmother's generations. However, two maternal uncles (III-2 and III-3, Figure 1a) died perinatally and the maternal grandmother had four male siblings, two of whom (II-2 and II-3, Figure 1a) died shortly after birth. The other two (II-4 and II-5, Figure 1a), who were epileptic, died in childhood. A maternal aunt (III-4, Figure 1a) was also stillborn for unknown reasons.

The proband (IV-1) was born at term by normal vaginal delivery weighing $3.1 \mathrm{~kg}$ and was noted to have a left congenital cataract. He presented with focal seizures at the age of 4 weeks. On examination, no other abnormality was noted apart from the cataract. His genitalia were normal. At 4 months, he developed infantile spasms and an electroencephalogram (EEG) confirmed hypsarrhythmia (Figure 2a). He was also found to have gallstones. At the age of five, he is profoundly delayed in all areas of development, is unable to feed orally and has remained refractory to anticonvulsant therapy. He continues to have daily seizures in the form of myoclonic jerks and occasional tonic-clonic

${ }^{1}$ Department of Genetics and Molecular Pathology, Neurogenetics Laboratory, SA Pathology, Adelaide, Australia; ${ }^{2}$ Department of Paediatrics, University of Adelaide, Adelaide, Australia; ${ }^{3}$ Clinical Genetics Unit, Birmingham Women's Hospital, Edgbaston, Birmingham, UK; ${ }^{2}$ Department of Paediatric Neurology, Birmingham Children's Hospital, Birmingham, UK; 5 Department of Radiology, Birmingham Children's Hospital, Birmingham, UK; ${ }^{6}$ School of Molecular and Biomedical Science, University of Adelaide, Adelaide, Australia

*Correspondence: Dr J Morton, Clinical Genetics Unit, Birmingham Women's Hospital, Edgbaston, Birmingham B15 2TG, UK.

Tel: +44 121627 2630; Fax: +44 121627 2618; E-mail: jenny.morton@bwhct.nhs.uk

7These authors contributed equally to this work.

Received 13 January 2009; revised 16 July 2009; accepted 16 July 2009; published online 9 September 2009 


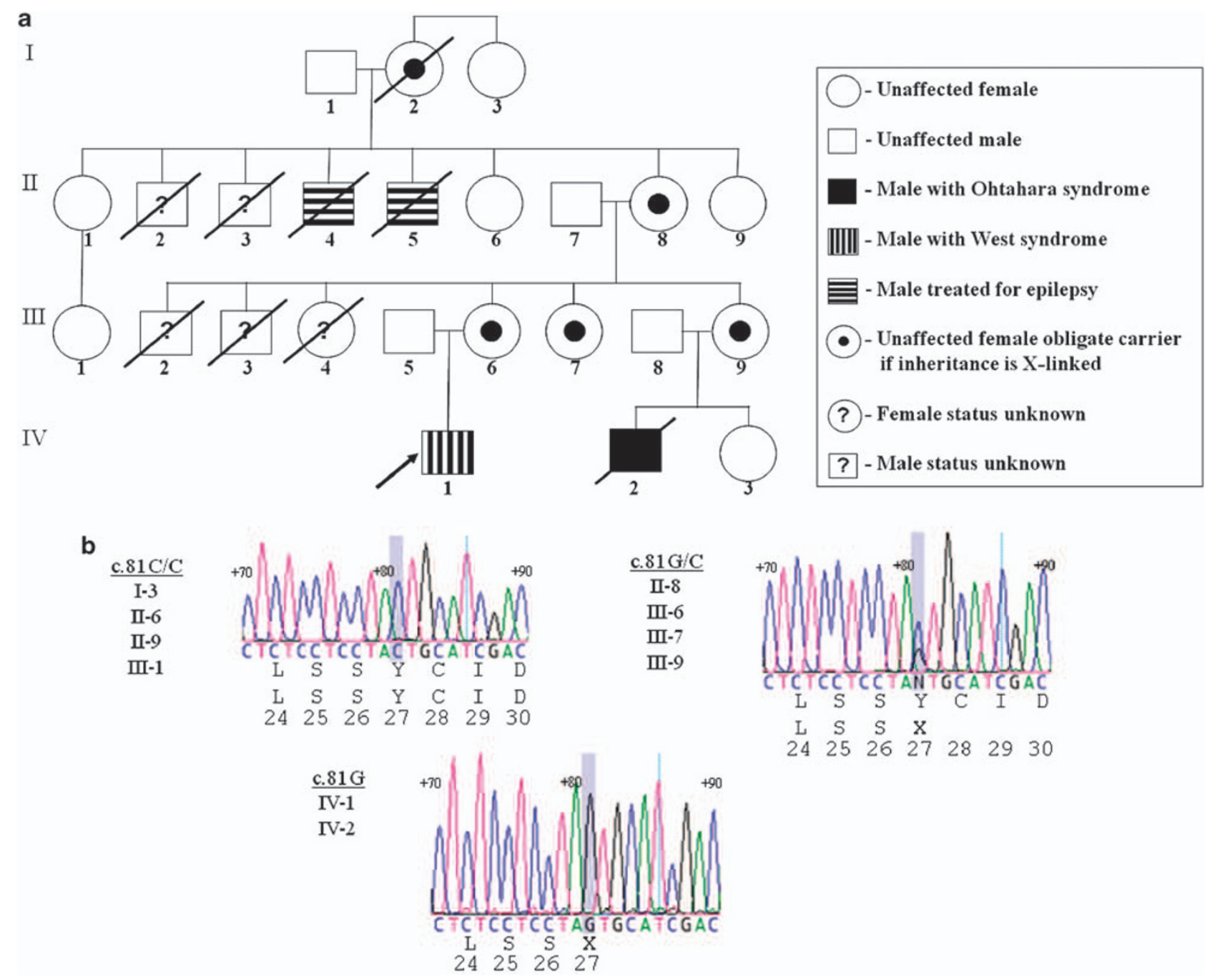

Figure 1 (a) The pedigree is compatible with an X-linked mode of inheritance. Affected males (IV-1, IV-2) and obligate carrier females (I-2, II-8, III-6 and III-9) are shown, with the proband (IV-1) designated by an arrow. Individuals II-4 and II-5 are presumed to have been affected. (b) Representative sequence traces are shown for all of the individuals tested for $A R X$ mutations, homozygous $A R X \mathrm{c} .81 \mathrm{C} / \mathrm{C}$ wt females (I-3, II-6, II-9, III-1), heterozygous $A R X \mathrm{c} .81 \mathrm{G} / \mathrm{C}$ carrier females (II-8, III-6, III-7, III-9) and hemizygous $A R X$ c.81G males (IV-1, IV-2).

fits. The most recent EEG shows a severe epileptic encephalopathy with subclinical status epilepticus in sleep and frequent myoclonic jerks associated with general discharges when awake (data not shown).

Individual IV-2 was a male infant born at 42 weeks by emergency Caesarian section for failure to progress, but did not require resuscitation. Birth weight was $3.54 \mathrm{~kg}$. He presented with tonic-clonic seizures and myoclonic jerks on day five. An EEG at 3 weeks was grossly abnormal showing a burst-suppression pattern consistent with a diagnosis of OS (Figure 2b), a severe early infantile epileptic encephalopathy (EIEE). By 5 months of age, he had axial hypotonia and hypertonia of all four limbs. His genitalia were normal. He made no developmental progress, was visually unaware and died aged 8 months from intractable seizures.

Cytogenetic analysis and extensive metabolic investigations were normal in both boys. Magnetic resonance imaging (MRI) of the brain was performed on both patients (Figure $3 a$ and $b$ ). The MRI brain scan of patient IV-1 at the age of 5 months showed mild cerebral atrophy, with no evidence of pachygyria or lissencephaly and normal myelination. A repeat MRI scan at the age of 21 months (Figure 3a) showed slight progression of the atrophy, an atrophic posterior corpus callosum (arrows on right image of Figure 3a), abnormal white matter signal for age and positional plagiocephaly. The MRI brain scan of patient IV-2 at the age of 4 weeks (Figure 3b) showed a normal gyral pattern with no evidence of pachygyria or lissencephaly and a hypoplastic corpus callosum (arrows on right image of Figure $3 \mathrm{~b}$ ) with absent rostrum, splenium and posterior body.

\section{Molecular analysis}

ARX mutation analysis. After the birth of IV-1 and having reviewed the family history (Figure 1a), X-linked recessive inheritance seemed likely and the possibility of an $A R X$-related disorder was explored. $A R X$ gene mutation analysis was performed, as described earlier. ${ }^{1}$ Each exon of the $A R X$ gene (including $\sim 30 \mathrm{bp}$ of flanking intronic sequence) was amplified by PCR and sequenced on an automated capillary sequencer (ABI 3100, Applied Biosystems, Foster City, CA, USA) and the sequence compared with the $A R X$ reference sequence (NM_139058) using SeqMan module of the Lasergene DNA and protein analysis software package (DNAStar Inc., Madison, WI, USA).

Generation of an $A R X \quad c .81 C>G$ expression construct. $p C M V-M y c-A R X$ wild-type (wt) vector was obtained ${ }^{7}$ and the c.81C $>$ G mutation was introduced by site-directed mutagenesis using the QuikChange site-directed mutagenesis kit (Stratagene, Cedar Creek, TX, USA) following manufacturer's instructions and using the following mutagenic primer: 5'-CAA CTT TGC TCT CCT CCT AgT GCA TCG ACA GCA TCC TGG G-3'. The entire open reading frame of the construct was then sequenced to ensure no other mutations had been introduced.

Transfection studies and cell culture. HEK-293T cells were transfected with $1 \mu \mathrm{g}$ of either $p C M V-M y c-A R X$ wt or $c .81 C>G$ vector and cultured using earlier described techniques. ${ }^{7,8}$ 


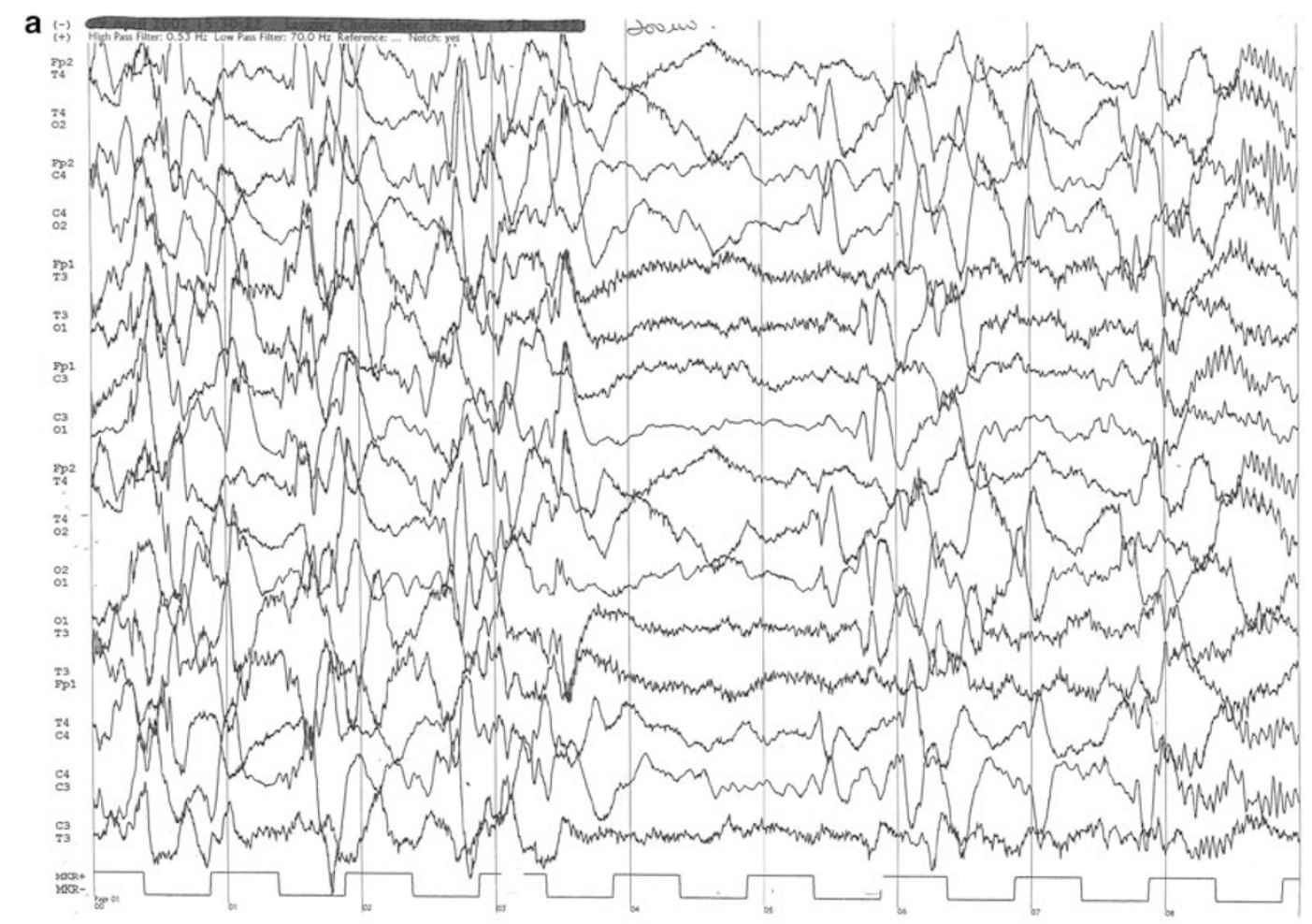

b

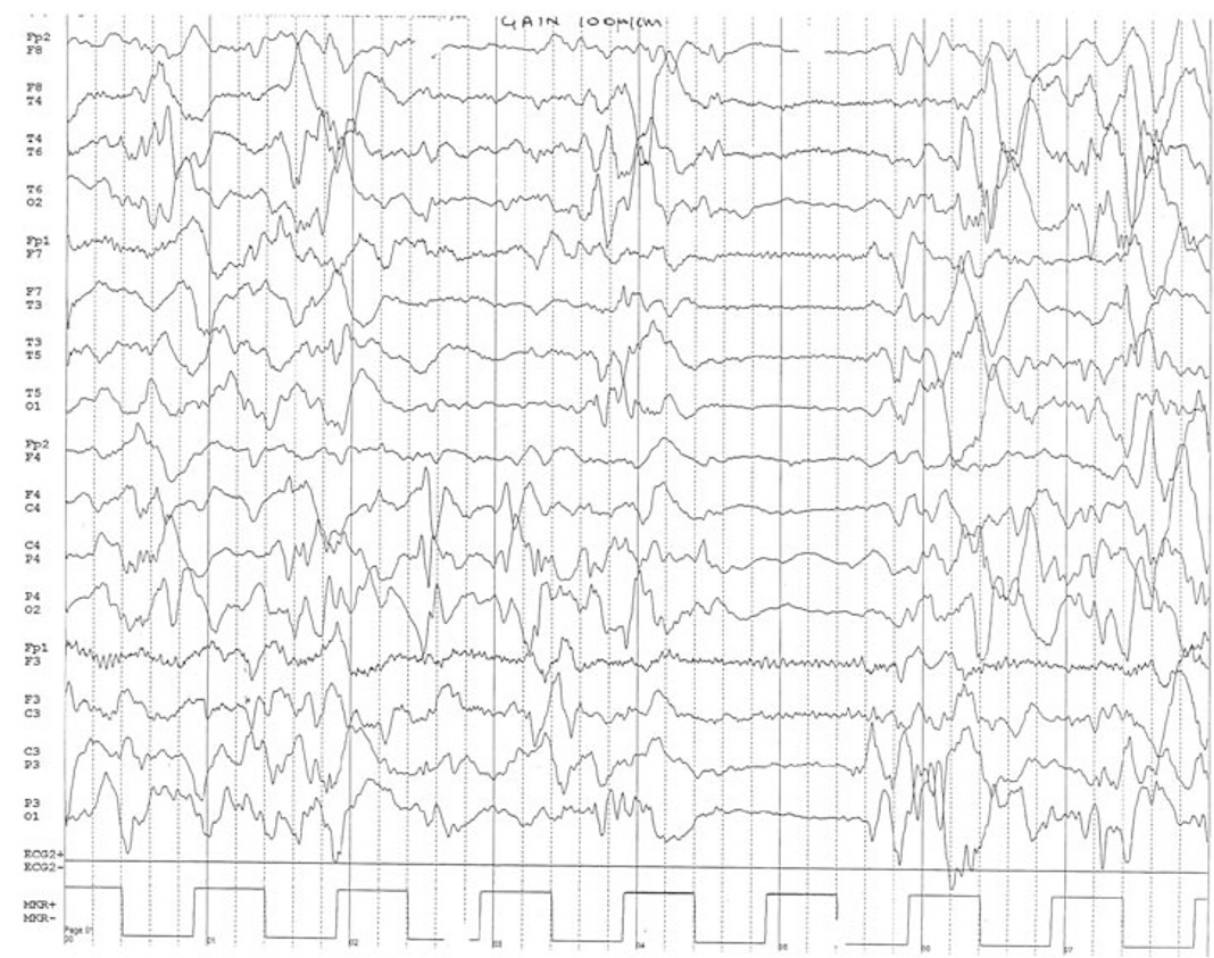

Figure 2 (a) EEG of individual IV-1 showing a hypsarrhythmia pattern with short periods of suppression. (b) EEG of individual IV-2 is grossly abnormal showing a burst-suppression pattern, consistent with a diagnosis of OS.

ARX polyclonal antibody production. We produced a polyclonal antibody against a small peptide based on p.161_179 of mature human ARX (KISQAPQVSISRSKSYREN). The peptide was synthetically manufactured (Thermo Fisher Scientific, Waltham, MA, USA) and conjugated to diphtheria toxoid. Polyclonal antibodies were produced by immunizing cross-bred Merino sheep with four injections of $2 \mathrm{mg}$ conjugated peptide in adjuvant at three-weekly intervals (IMVS, Adelaide, SA, Australia). Serum was collected and antibodies were then purified by protein $G$ affinity chromatography.

ARX protein analysis. 293T cells transfected with $1 \mu \mathrm{g} p C M V-M y c-A R X$ constructs were harvested and protein extracted for western immunoblot. 

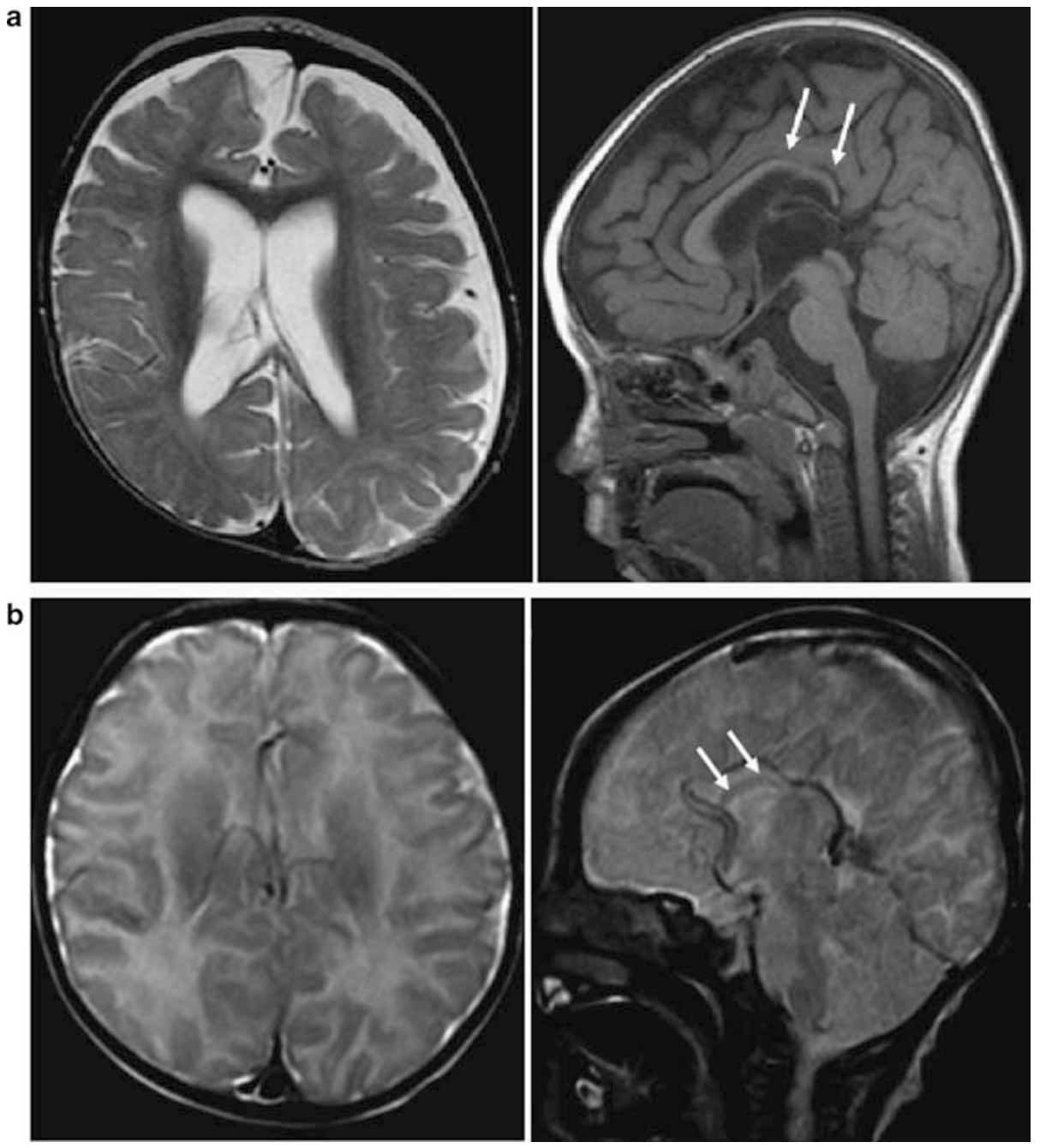

Figure 3 (a) MRI brain scan of individual IV-1 at 21 months: axial T2 and sagittal T1 weighted images showing no lissencephaly, but cerebral atrophy, abnormal white matter signal, an atrophic posterior corpus callosum (arrows) and plagiocephaly. (b) MRI scan of individual IV-2 at 4 weeks: Axial and sagittal T2 weighted images showing normal gyral pattern and a hypoplastic corpus callosum (arrows).

Lysates were then subjected to SDS-PAGE, transferred to nitrocellulose and analyzed by immunoblotting. The Sheep $\alpha$-ARX polyclonal antibody was diluted $1 / 2000$ and detected by a Donkey $\alpha$-Sheep IgG-HRP-conjugated secondary antibody (Millipore, Billerica, MA, USA) diluted 1/2000. The membrane was stripped in $0.2 \mathrm{M} \mathrm{NaOH}$ for $5 \mathrm{~min}$ after ARX detection to enable immunoblotting of the same membrane with the cMYC antibody. The mouse $\alpha$-cMYC monoclonal antibody (Santa Cruz Biotechnology, Santa Cruz, CA, USA) was diluted $1 / 1000$ and was detected by an $\alpha$-mouse IgG-HRPconjugated secondary antibody (Dako, Glostrup, Denmark) diluted 1/2000. Signal was detected through chemiluminescence.

\section{RESULTS}

We identified a novel $A R X$ nucleotide change, c. $81 \mathrm{C}>\mathrm{G}$ in exon 1 of the gene in patient IV-1. Subsequently, mutation analysis on DNA extracted from peripheral blood from family members I-3, II-6, II-8, II-9, III-1, III-6, III-7, III-9 and IV-2 (using Guthrie card sample) was performed. Both affected males (IV-1 and IV-2) were found to be hemizygous for the c.81C $>\mathrm{G}$ mutation (Figure $1 \mathrm{~b}$ ), whereas their mothers (III-6, III-9), aunt (III-7) and maternal grandmother (II-8) were shown to be heterozygous carriers (Figure 1b). The two maternal great aunts (II-6 and II-9) were found to be homozygous for the wt allele (Figure 1b). The great grandmother (I-2) was inferred to be a carrier, whereas her sister (I-3) and granddaughter (III-1) are homozygous for the wt allele.

The $c .81 \mathrm{C}>\mathrm{G}$ nucleotide change is predicted to cause a replacement of the tyrosine (p.Y27) codon (UAC) with a premature termination codon (PTC; UAG) leaving the ARX protein only 26 amino-acids long (Figures $1 \mathrm{~b}$ and $\overline{4} \mathrm{a})$. As such, this p.Y27X change is highly likely to be deleterious and, thus, considered to be the mutation responsible for the observed phenotype in this family.

An N-terminally truncated ARX was detected in lysates of HEK-293T cells transiently transfected with a vector ( $p C M V-M y c)$ that over-expresses the c.81C $>\mathrm{G}$ mutant form of $A R X$ (Figure 4). This truncated ARX, most likely ARX p.M41_C562, is detected by an ARX polyclonal antibody, but not by a cMYC antibody that detects the N-terminal MYC tag. As there is 10 times more protein extract in the c.81C>G lane and the truncated ARX protein is detected at approximately half the level of the wt, there is approximately less than 1/20th the amount of the re-initiation product compared with wt. We predict that the MYC-tagged ARX p.S26 peptide is degraded, and as such could not be detected by the cMYC antibody. 
a
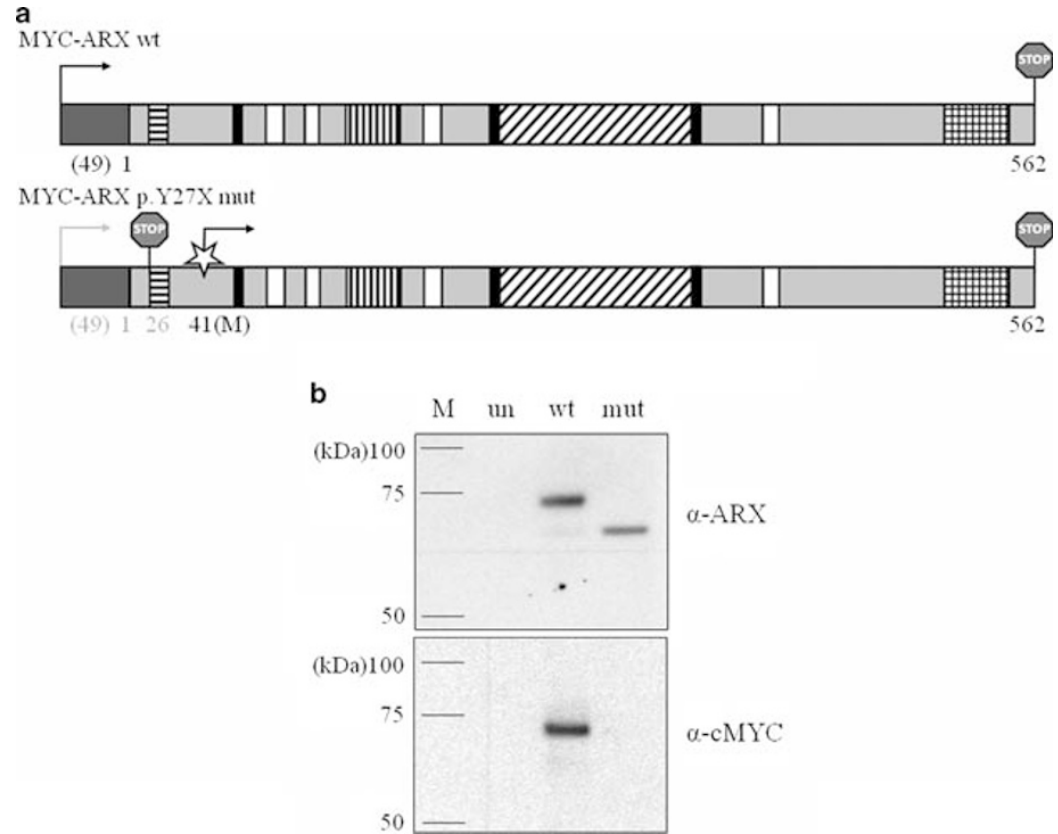

Figure 4 (a) Schematic of ARX wt and p.Y27X mutant myc-tagged ARX proteins (adapted from Ref. ${ }^{7}$ ). Shaded dark grey is the 13 amino acids (aa) of the MYC tag with a further 26aa of leader peptide before the p.M1 of ARX. The total number of aa added by the MYC tag and leader sequence is 49 . The ARX protein (grey) has aa residue numbers in black below each schematic and contains the following domains: OP, octapeptide domain (horizontal hatch); NLS13, nuclear localization sequence (black); pA1-4, polyalanine tracts (white); acidic domain (vertical hatch); homeodomain (diagonal hatch) and OAR Aristaless domain (cross-hatched). Initiation of translation is indicated by an arrow above the protein schematics. A premature $\mathrm{N}$-terminal termination codon is introduced by the $c .81 \mathrm{C}>\mathrm{G}$ mutation, as represented in the lower protein schematic. The star indicates the predicted residue (p.M41) at which re-initiation of translation most likely occurs. The predicted protein sizes that result from over-expression from a CMV promoter in transiently transfected HEK-293T are MYC-ARXwt-611 aa (62 kDa), MYC-ARX(p.M1_S26)—75 aa (4kDa), ARX(p.M41_C562)—522 aa (53.7 kDa). (b). SDS-PAGE western immunoblot of pCMV-Myc-ARX transfected HEK-293T cell lysates. Lanes were loaded as follows: M-marker $10 \mu \mathrm{l}$ of precision plus protein standards, un-20 $\mu \mathrm{g}$ of mock transfected cells, wt $-2 \mu \mathrm{g}$ of $p C M V-M y c-A R X w t$ transfected cells, mut $-20 \mu \mathrm{g}$ of $p C M V-M y c-A R X C .81 C>G$ transfected cells. The same membrane was first probed with the ARX polyclonal antibody, stripped, then re-probed with the CMYC antibody.

\section{DISCUSSION}

The $A R X$ mutation identified in this family is predicted to cause premature protein truncation. Other $A R X$ mutations predicted to cause an early truncation of the ARX protein (EX2-5del, c.196+2T > C, c.232G > T; EX1-2.1del; reviewed in Ref. ${ }^{4}$ ) have been described. Interestingly, virtually all of these cause the X-linked lissencephaly with ambiguous genitalia (XLAG) phenotype, which represents the severe malformation end of the phenotypic spectrum of $A R X$-associated disorders. ${ }^{9,10}$ All of these mutations can also be considered $A R X$ null mutations, which is an assumption supported by the original discovery of the human XLAG mutations that mimic the Arx mouse knockout model. ${ }^{3}$ We predict that the $c .81 \mathrm{C}>\mathrm{G} /$ p.Y27X mutation is also akin to a null mutation. The resultant p.Y27X ARX protein would lack all of its functional domains, including the octapeptide domain (aa 27-34), and would either not be made, be non-functional or rapidly degraded. In our family, although minor brain abnormalities were visible on the MRI brain scans in the affected individuals, there is a striking lack of the hallmark lissencephaly and ambiguous genitalia associated with the XLAG phenotype. After detecting an N-terminally truncated ARX protein generated from the over-expression of the p.Y27X mutant, we speculated that $A R X$ mRNA translation re-started at a more downstream AUG/Methionine codon. Only one such in-frame codon, at position c.121_123 of ARX, fulfilled this role, suggesting that the N-terminally truncated ARX protein consists of p.M41_C562. Re-initiation of mRNA translation is a known, although still poorly understood, phenomenon (for review see Ref. ${ }^{11}$ ). Translation re-initiation may explain the relatively 'mild' non-malformation phenotype observed in this family, consistent with the observations made in other diseases. ${ }^{12}$ However, this explanation is subject to the proposition that the in vivo c.81C $>\mathrm{G}$ containing $A R X$ mRNA escapes degradation by the nonsense-mediated decay (NMD) pathway. We predict that NMD partially degrades the c.81C $>\mathrm{G} A R X$ mRNA, as is seen with other PTC containing mRNAs, ${ }^{13}$ and is most likely protected from complete degradation because of the proximity of the PTC to the translation initiation codon. ${ }^{14}$ Unfortunately, we were unable to test $A R X$ mRNA or protein expression in vivo because of the lack of a relevant $A R X$ expressing tissue source from the patients. Provided that $A R X$ mRNA translation does re-initiate at codon c.121_123 (aa 41 of wt ARX), such an N-terminally truncated protein would completely lack the octapeptide domain (aa 27-34). This domain is an important transcription co-repression domain of ARX and is involved in binding to the Groucho/transducin-like enhancer of split co-factor proteins. ${ }^{8}$ The p.L33P mutation of the octapeptide domain is known to give a nonsyndromic phenotype in the MRX54 family, ${ }^{2}$ but perhaps a more severe phenotype would have been observed if the entire octapeptide was absent.

In summary, we speculate that the lack of a severe malformation phenotype observed for these patients could be explained by a partially functioning, N-terminally truncated ARX protein (p.M41_C562). This truncated ARX protein might be less abundant than the wt (Figure 4), probably because of partial NMD degradation of the PTC containing $A R X$ mRNA and the inefficiency of translation re-initiation from the c.121_123 AUG codon. 
Another possible mechanism behind the partial phenotypic rescue of these patients is RNA editing of the c.81C $>\mathrm{G}$ containing $A R X$ mRNA, which would allow translation of a full-length ARX mRNA (reviewed in Ref. ${ }^{15}$ ). However, this could not be tested because of the unavailability of a suitable patient material.

Investigations did not reveal an underlying cause for the cataract or gallstones in patient IV-1, neither of which has been described earlier in patients with an $A R X$ gene mutation and the significance of these features remains unclear.

OS is an EIEE with the earliest known age of onset for any agedependant epileptic syndrome, often presenting within days of birth or even prenatally. ${ }^{16}$ OS is characterized by frequent minor generalized seizures and 'burst suppression' on the EEG. 'Bursts' are high amplitude slow activities, often with sharp waves or spikes, interspersed with periods of 'suppression' with little or no activity. The seizures are intractable and are co-morbid with severe psychomotor retardation. Prognosis is poor with about one in three patients dying before the second year of life. OS can be distinguished from other neonatal epileptic encephalopathies by the disease's progression during development. ${ }^{16}$ OS progresses into WS in $75 \%$ of cases at approximately 3-4 months of age. After this, 59\% of WS cases develop into Lennox-Gastaut syndrome (LGS), ${ }^{17}$ distinguishing it from early myoclonic encephalopathy, another EIEE, which neither progress into WS nor LGS. ${ }^{16}$ The aetiology of OS is heterogeneous, ${ }^{16}$ and can be caused by structural abnormalities such as hemimegencephaly, porencephaly, hydrocephaly and lissencephaly and includes syndromes such as Aicardi syndrome. In addition, a cryptogenic non-malformation form occurs. Similar to mental retardation, there is a $30 \%$ excess of males with OS, indicative of the involvement of genes located on the $\mathrm{X}$ chromosome, ${ }^{18}$ and an $A R X$ polyalanine expansion mutation has recently been described as a cause of OS in two out of three sporadic male cases. ${ }^{19} A R X$ is expressed in GABAergic neurons, and Kato et $a l^{19}$ hypothesized that dysfunction of the GABAergic system is critical to the neuropathology of EIEE and WS. In addition, heterozygous mutations in the STXBP1 gene seem to cause OS in both males and females. ${ }^{20}$

OS and XLAG have many features in common such as early onset during infancy, frequent intractable seizures, abnormal burst-suppression EEG patterns, hypotonia, impaired motor function and profound developmental delay. Mutations in ARX have been reported to account for up to $94 \%$ of XLAG ${ }^{9}$ and be responsible for X-linked WS. ${ }^{1}$ On the basis of our findings, we suggest that impaired function of the ARX protein can also result in OS. Perhaps this finding is not surprising given the clinical overlap between XLAG and OS, as well as the progression of most OS cases into WS. In addition, this family shows intrafamilial clinical heterogeneity, indicating the influence of different genetic and environmental background on the clinical expression of a given $A R X$ gene mutation. Specifically in this case, we suggest that subtle differences in genetic background and environment affected the extent to which NMD or translation re-initiation occurred in the two affected individuals, leading to the different clinical manifestations. To our knowledge, this is only the second report of an ARX gene mutation causing $O S$ and the first with an inherited mutation. Interestingly, in the cases reported by Kato et al, ${ }^{19}$ both had a 33-bp duplication in exon 2 of $A R X$ rather than a protein truncating mutation. This supports the conclusion that modified function rather than complete loss of function of the ARX protein may be the cause of OS in our family. This mutation represents the first ARX protein truncation mutation causing non-malformation phenotype and points towards the complexities of the interplay between the mutation, molecular mechanism and the genetic background. It also reminds us to exercise caution when drawing conclusions about genotypephenotype correlation. We suggest that $A R X$ screening should be considered in affected males with OS.

\section{ACKNOWLEDGEMENTS}

We thank the members of the family for their cooperation and help. We also thank Cheryl Shoubridge for sharing the $p C M V-M y c-A R X w t$ construct and the sheep polyclonal anti ARX antibody. This study was supported by research grants from the NH\&MRC awarded to JG.

1 Strømme P, Mangelsdorf ME, Shaw MA et al: Mutations in the human ortholog of Aristaless cause X-linked mental retardation and epilepsy. Nat Genet 2002; 30: 441-445.

2 Bienvenu T, Poirier K, Friocourt G et al: ARX, a novel Prd-class-homeobox gene highly expressed in the telencephalon, is mutated in X-linked mental retardation. Hum Mol Genet 2002; 11: 981-991.

3 Kitamura K, Yanazawa M, Sugiyama $\mathrm{N}$ et al: Mutation of ARX causes abnormal development of forebrain and testes in mice and $X$-linked lissencephaly with abnormal genitalia in humans. Nat Genet 2002; 32: 359-369.

$4 \mathrm{Gecz}$ J, Cloosterman D, Partington M: ARX: a gene for all seasons. Curr Opin Genet Dev 2006; 16: 308-316.

5 Colombo E, Galli R, Cossu G, Gecz J, Broccoli V: Mouse orthologue of ARX, a gene mutated in several X-linked forms of mental retardation and epilepsy, is a marker of adult neural stem cells and forebrain GABAergic neurons. Dev Dyn 2004; 231: 631-639.

6 Poirier K, Van Esch H, Friocourt G et al: Neuroanatomical distribution of ARX in brain and its localisation in GABAergic neurons. Brain Res Mol Brain Res 2004; 122 : 35-46.

7 Shoubridge C, Cloosterman D, Parkinson-Lawerence E, Brooks D, Gécz J: Molecular pathology of expanded polyalanine tract mutations in the Aristaless-related homeobox gene. Genomics 2007; 90: 59-71

8 McKenzie 0, Ponte I, Mangelsdorf M et al: Aristaless-related homeobox gene, the gene responsible for West syndrome and related disorders, is a Groucho/transducin-like enhancer of split dependent transcriptional repressor. Neuroscience 2007; 146: 236-247.

9 Kato M, Das S, Petras $\mathrm{K}$ et al: Mutations of ARX are associated with striking pleiotropy and consistent genotype-phenotype correlation. Hum Mutat 2004; 23: 147-159.

10 Bhat SS, Rogers RC, Holden KR, Srivastava AK: A novel in-frame deletion in ARX is associated with lissencephaly with absent corpus callosum and hypoplastic genitalia. Am J Med Genet A 2005; 138: 70-72.

11 Kozak M: Pushing the limits of the scanning mechanism for initiation of translation. Gene 2002; 299: 1-34.

12 Paulsen M, Lund C, Akram Z, Winther JR, Horn N, Moller LB: Evidence that translation reinitiation leads to a partially functional Menkes protein containing two copperbinding sites. Am J Hum Genet 2006; 79: 214-229.

13 Maquat LE: Nonsense-mediated mRNA decay: splicing, translation and mRNP dynamics. Nature Rev Mol Cell Biol 2004; 5: 89-99.

14 Silva AL, Ribeiro P, Inácio A, Liebhaber SA, Romão L: Proximity of the poly(A)-binding protein to a premature termination codon inhibits mammalian nonsense-mediated mRNA decay. RNA 2008; 14: 563-576.

15 Mattick JS, Mehler MF: RNA editing, DNA recoding and the evolution of human cognition. Trends Neurosci 2008; 31: 227-233.

16 Ohtahara S, Yamatogi Y: Ohtahara syndrome: with special reference to its developmental aspects for differentiating from early myoclonic enecephalopathy. Epilepsy Res 2006; 70 (Suppl 1): S58-S67.

17 Yamatogi Y, Ohtahara S: Early-infantile epileptic encephalopathy with suppressionbursts. Ohtahara syndrome: its overview referring to our 16 cases. Brain Dev 2002; 24: 13-23.

18 Djukic A, Lado FA, Shinnar S, Moshe SL: Are early myoclonic encephalopathy (EME) and the Ohtahara syndrome (EIEE) independent of each other? Epilepsy Res 2006; 70 (Suppl 1): S68-S76.

19 Kato M, Saitoh S, Kamei A: A longer polyalanine expansion mutation in the ARX gene causes early infantile epileptic encephalopathy with suppression-burst pattern (Ohtahara syndrome). Am J Hum Genet 2007; 81: 361-366.

20 Saitsu H, Kato M, Mizuguchi T et al: De novo mutations in the gene encoding STXBP1 (MUNC18-1) cause early infantile epileptic encephalopathy. Nat Genet 2008; 40: 782-788. 